\title{
chameleon: Delivering Improved Sample Preparation for Single Particle CryoEM
}

\author{
Michele Darrow, John Moore, Klaus Doering, Herve Vandekerckhove, Paul Thaw and Russell King
}

SPT Labtech, Melbourn, England, United Kingdom

Sample preparation for cryo electron microscopy (cryoEM) now represents the bottleneck in the process to high-resolution structure determination. The basic premise of cryoEM sample preparation was pioneered over 30 years ago by Dubochet and colleagues and largely has not been updated since. This process, while revolutionary in its' time, is now seen as difficult, wasteful of sample and microscope time, and requiring both skill and luck to produce a high-quality grid [1].

Additionally, technical requirements of the sample preparation process have shifted with the maturation of the cryoEM field. As more imaging facilities are founded, the need for automation of the process increases, and the cost of screening poor grids is magnified. At the same time, new interests in the field, such as integrated cellular proteomics, time-resolved imaging, and microED, require a sample preparation system that is flexible enough to accommodate a wide range of varied samples and sample requirements.

Finally, the air-water interface (AWI) has been recently recognized as a hostile environment for specimens. The negative consequences of samples spending time at the AWI during sample preparation can include partial or full denaturation and the adoption of a preferred orientation [2]. These effects have been identified as time-dependent, with partial mitigation through faster sample preparation [3].

The chameleon system, based on Spotiton [4,5] represents the next generation of sample preparation devices for cryoEM. The system is composed of a blot-free, pico-litre dispensing instrument, with fully automated handling of self-wicking grids and a workflow-based software to guide the user through grid preparation.

A minimum of $5 \mu \mathrm{l}$ of sample is loaded into the instrument of which $3 \mu \mathrm{l}$ is aspirated into the dispenser. Sample application occurs "on the fly" as grids pass in front of the dispenser. Each stripe placed onto the grid is $\sim 6 \mathrm{nl}$ of sample, meaning many grids can be prepared from a single $3 \mu 1$ aspiration.

An integrated glow discharge unit and multiple onboard cameras to monitor the process together provide control of and ice thickness screening without the use of an electron microscope, reducing the time and cost normally associated with the screening step.

The standard plunge profile allows grids to be prepared at times between $101 \mathrm{~ms}$ and 2,500 ms, while the high-speed plunge profile provides a dispense-to-plunge time of $54 \mathrm{~ms}$. In both cases, visual feedback is provided, allowing the user to screen the grids just $50 \mathrm{~ms}$ (40 $\mathrm{ms}$ in the case of the high-speed plunge profile) prior to entering the ethane.

chameleon systems provide automation of the sample preparation process, visual quality control without the need for an electron microscope, and guiding software. In addition, the fast dispense-to-plunge times can address issues caused by the AWI enabling cutting-edge research.

Here, results will be discussed to showcase concepts and exciting structural outcomes from in-house testing, a beta-user program and early adopter sites. 
A

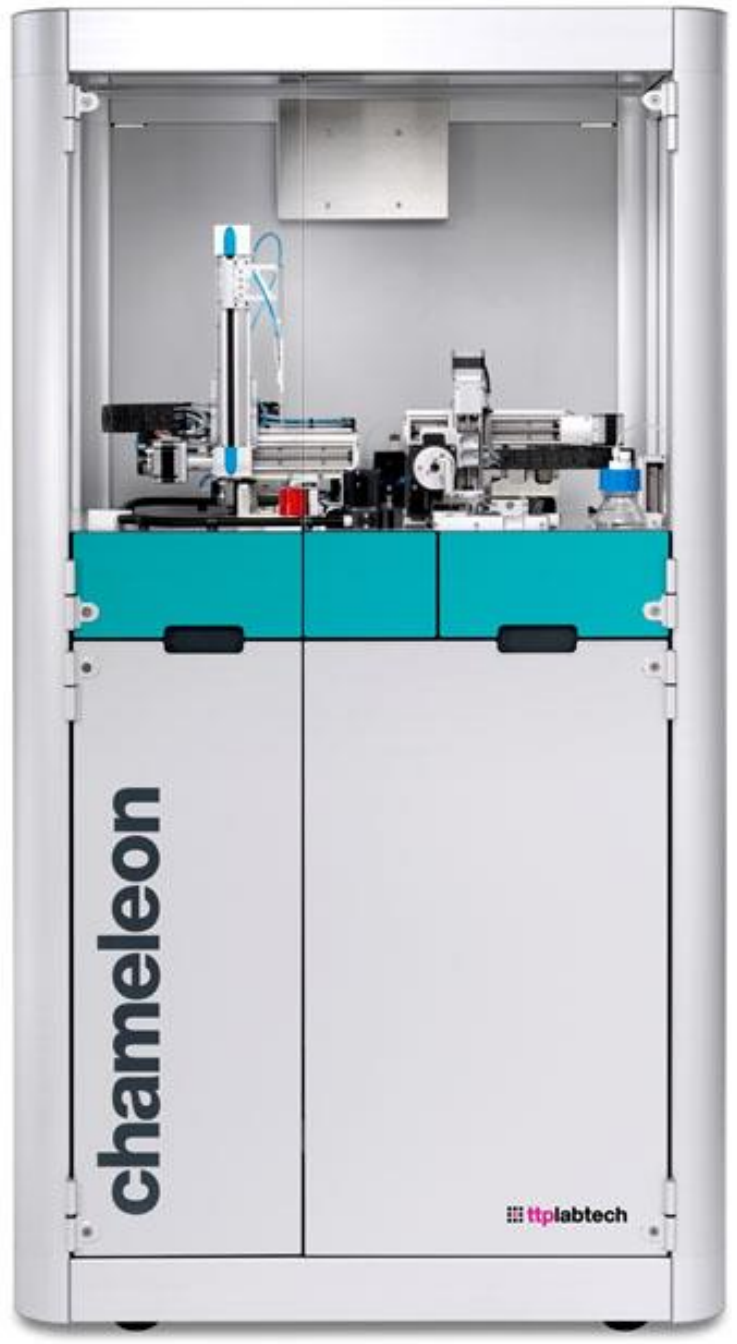

$\mathbf{B}$

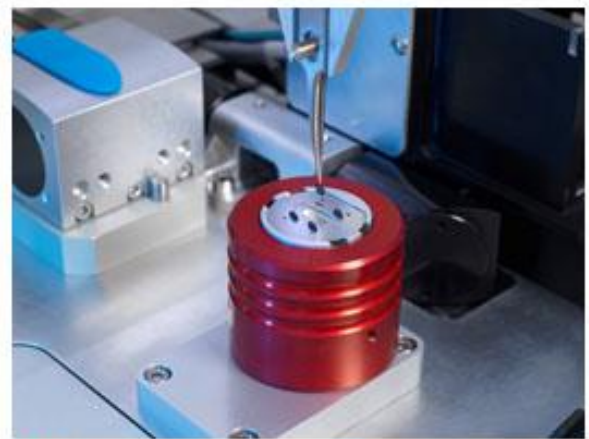

C

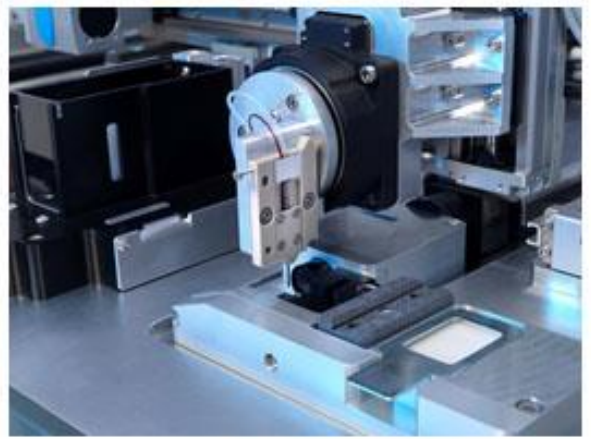

D

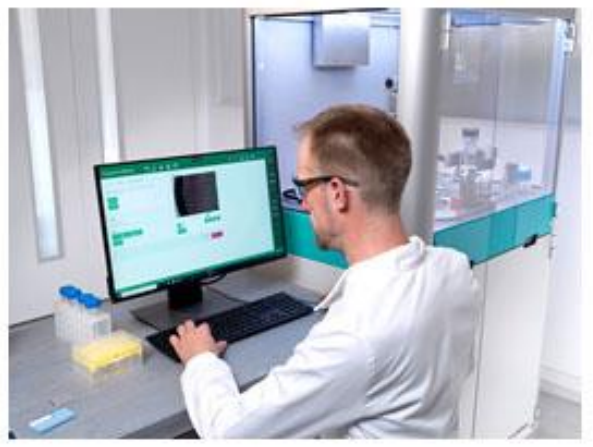

Figure 1. The chameleon system for improved sample preparation in single particle cryoEM. (A) The chameleon system. (B) The grid holder with automatic grid pick-up. (C) The dispenser and dispenser set-up area, including onboard camera for visualising sample dispense. (D) Guided software workflows and automated detection of appropriate wicking times aid the user.

\section{References}

[1] B Carragher, et al. Journal of Microscopy 276 (2019), p. 39-45.

[2] RM Glaeser. Current Opinions in Colloid \& Interface Science 34 (2018), p. 1-8.

[3] A Noble, et al. Nature Methods 15 (2018), p. 793-795.

[4] V Dandey, H Wei, et al. Journal of Structural Biology 202 (2018), p. 161-169.

[5] H Wei, V Dandey, et al. Journal of Structural Biology 202 (2018), p.170-174. 